\title{
Formation of Nanosystems under Near-Equilibrium Copper Condensation in an Ultrapure Inert Medium
}

\author{
V. I. Perekrestov*, A. S. Kornyushchenko, and Yu. A. Kosminskaya \\ Sumy State University, Sumy, 40007 Ukraine \\ *e-mail:perv@phe.sumdu.edu.ua \\ Received October 31, 2007
}

\begin{abstract}
Mechanisms of the self-organization of nanosystems at the condensation of the extremely weak steady flows of copper vapors obtained in magnetron sputtering in an ultrapure argon are studied. It is shown that the freeenergy minimization near equilibrium condensation creates prerequisites for the self-organization of statistically homogeneous layers of nanoclusters and determines the transition to the formation of nanosystems in the form of fractal networks.
\end{abstract}

PACS numbers: 68.55.-a, 81.07.Bc, 81.15.Cd

DOI: $10.1134 / \mathrm{S} 0021364007240046$

The aspects of the self-organization of the statistically uniform functional nanosystems are of key importance in the development of nanotechnologies. The monodisperse island layers are most frequently produced under the near-equilibrium Stranski-Krastanov conditions. In this case, the self-organization of statistical uniformity follows from the minimization of the energy of elastic stresses arising in the wetting layer [1]. However, the possibility of the implementation of the metal monodisperse island nanosystems based on the Volmer-Weber mechanism has not as yet been studied. It is generally agreed that the nucleation of metal condensates under the Volmer-Weber conditions has the stages of the formation of a statistically nonuniform layer of supercritical nuclei, the coalescence of the islands, the formation of the channels, and the subsequent transition to a continuous film [2]. We note that all the above stages are accompanied by an intense secondary nucleation, which, in turn, is a consequence of a considerably high supersaturation. The latter statement is based on the fact that, according to Volmer and Weber, the condensation mechanism has conventionally been studied in the deposition of metal vapors whose low volatility considerably complicates the implementation of the near-equilibrium conditions. The final result of the condensation of sufficiently supersaturated vapors is the formation of continuous polycrystalline or epitaxial layers [2].

On the example of the deposition of the extremely weak $\mathrm{Al}$ and $\mathrm{Ti}$ vapor flows, we identified the transition to the formation of highly porous three-dimensional structures in the form of nanocrystals weakly bound with each other $[3,4]$. However, no signs of the selforganization of an island structure monodispersity have been revealed in this case. More recently, it was found that the transition to three-dimensional highly porous formations is conditioned by the maximum approach to the phase equilibrium in the environment-condensate system [5]. In this case, the phase selectivity is observed together with the well-pronounced spatially distributed condensate growth $[6,7]$.

The condensate nucleation under the extremely low supersaturation conditions is based on the barrier nucleation, which is explained within the framework of nonequilibrium thermodynamics and described in kinetic theory $[8,9]$. Under similar conditions, the fluctuation mechanisms lead to the growth or reduction of subcritical nuclei; this behavior may result in the directional "diffusion along the size axis" to subcritical nuclei when supersaturation increases up to a certain value. The processes occurring in this case determine the ultimate minimization of the free energy, which may, in turn, cause the self-organization of an identical shape and size of the islands, as well as of a certain order in their mutual arrangement.

In the context of the above preface, the objective of this work is to reveal the self-organization conditions for copper nanosystems based on the near-equilibrium condensation under Volmer-Weber conditions.

\section{EXPERIMENTAL PROCEDURE}

The required technological conditions were implemented by the dc magnetron sputtering of copper at an Ar pressure of $10 \mathrm{~Pa}$. Argon was subjected to deep purification via the absorption of chemically active gases during the process of additional Ti sputtering [10]. The partial pressure of the chemically active residual gases (mainly $\mathrm{O}_{2}, \mathrm{H}_{2}$, and $\mathrm{N}_{2}$ ) was $8 \times 10^{-8} \mathrm{~Pa}$. Fresh $\mathrm{KCl}$ cleavages were used as the substrates. 

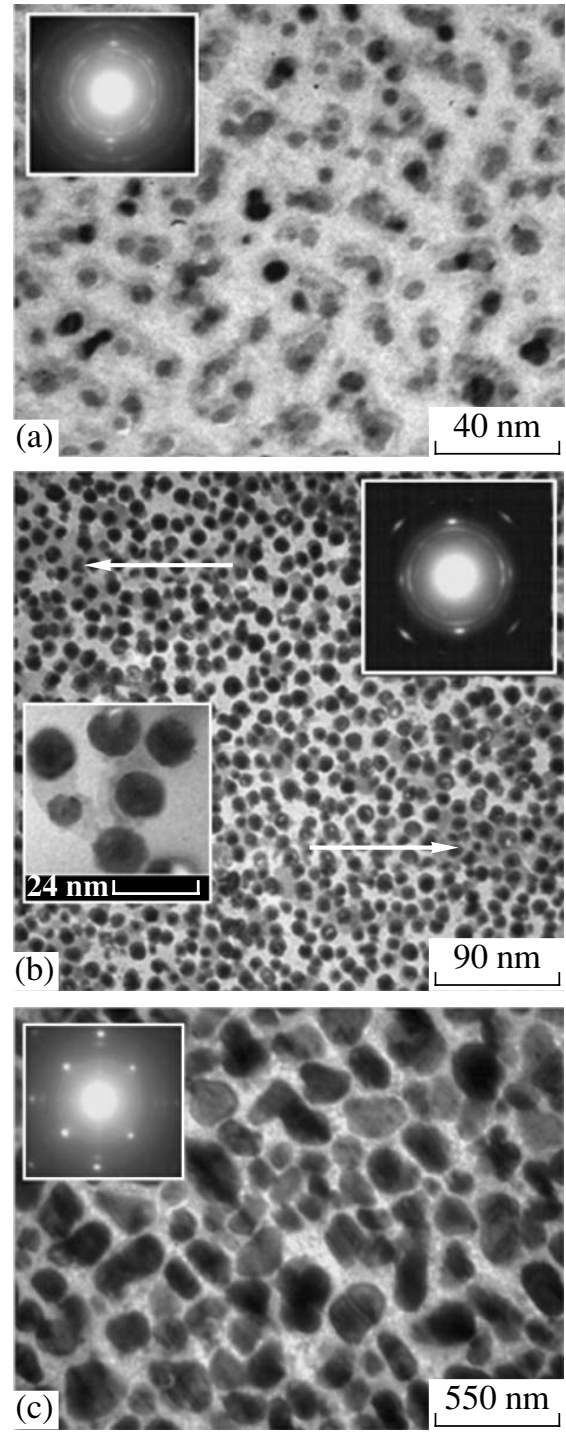

Fig. 1. Structure of the copper island condensates: $(a, b)$ $P_{w}=24 \mathrm{~W}$ and the deposition time is 8 and $12 \mathrm{~min}$, respectively; and (c) $P_{w}=28 \mathrm{~W}$, the deposition time is $5 \mathrm{~min}$.

The reduction of the free path length at the increased working gas pressures allowed us to average the energy of the ion-sputtered atoms [11], as well as to ensure the equiprobable arrival of the substance onto different facets of the growing crystals near the growth surface. In this case, the average energy of the ion-sputtered atoms is one order of magnitude higher than the energy of thermally evaporated atoms and is equal to about 5$8 \mathrm{eV}$ [11], which corresponds to a particle temperature of $\sim 38000 \mathrm{~K}$. Moreover, it is known $[12,13]$ that the incomplete thermal accommodation needs the energy of condensed atoms corresponding to a temperature above $6000 \mathrm{~K}$. Hence, the incomplete thermal accommodation enhances the reevaporation of adatoms and, thus, makes it possible to achieve near-equilibrium conditions at relatively low substrate temperatures. Since copper has a low volatility, this circumstance is important for the solution of the problem stated in this work. In this case, the maximum approach to the equilibrium was also stimulated by an increased substrate temperature $\left(T_{\mathrm{c}}=380^{\circ} \mathrm{C}\right)$ and a decrease in the discharge power $\left(P_{w}\right)$ to a value level at which the finite rate of supercritical nucleation can still be observed.

The island structures formed were strengthened by continuous amorphous carbon layers and then separated from the substrates by dissolving the latter in water. The structure and phase composition of condensates were studied by transmission electron microscopy and electron microdiffraction.

\section{EXPERIMENTAL RESULTS AND DISCUSSION}

Near-equilibrium nucleation is possible only on the active centers of the maximum energies of chemical bonds [14]. In this connection, we note that on the (100) alkali halide crystal, the anion vacancies $\left(\mathrm{Cl}^{-}\right)$, which are formed in the crystals irradiated by elementary particles [15], have a high activity. Many of these active centers may appear in our case when the $\mathrm{KCl}$ cleavage is exposed to the flux of secondary electrons emitted from the magnetron [11]. Since the spectrum of the maximum possible energies of chemical bonds is discrete, there is a critical energy of chemical bonds $E_{\mathrm{c}}$ below which the atom fixation on the growth surface is a highly improbable process. Obviously, as the supersaturation tends to zero, $E_{\mathrm{c}}$ increases and, in the limiting case, the spectrum of active centers may reduce to a single possible variant with a corresponding fixation of adatoms only on the anion vacancies.

An analysis of the structure of the island systems obtained under these extreme conditions showed that, at the initial stage of their formation, thin $\mathrm{Cu}$ layers are formed on the local substrate regions; then, nanoclusters nucleate on these layers (see Fig. 1a). It may be suggested that the local formation of the thin base layers occurs on the anion vacancy clusters, which, in essence, constitute an atomically rough potassium surface. Under these conditions, the formation of a wetting layer is not impossible.

The analysis of the electron diffraction pattern presented in Fig. 1a reveals two variants of the nucleation texture, where (011) and (001) $\mathrm{Cu} \|(001) \mathrm{KCl}$. In this connection, we note that the further growth of the thin layer in the directions normal to (011) and (001) $\mathrm{Cu}$ is very problematic, because the atoms in these planes in the semicrystal position have relatively low energies of the chemical bonds [14] or energies lower than $E_{\mathrm{c}}$.

To analyze the transition to the cluster formation, we consider the following expressions for the rate $J$ of barrier nucleation [8]:

$$
J \propto \exp \left[-b / \ln ^{2}(\zeta)\right] .
$$



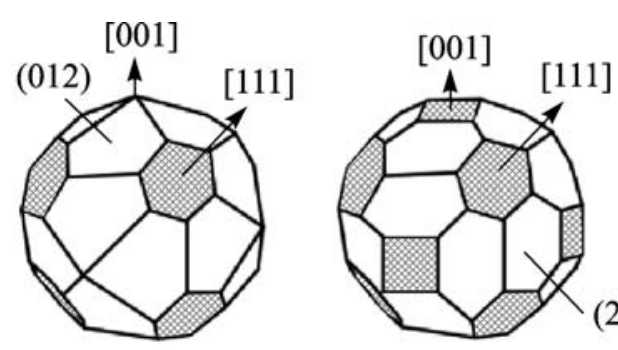

(210)

Fig. 2. Habituses of the clusters (light facets are built on the base of the (210) plane).

Here, $b=16\left(\alpha / k_{b} T\right)^{3} \sigma^{3} F / 3$, where $\alpha$ is the surface energy of a cluster, $\sigma$ is the surface area occupied by a single adatom, and $T$ is the temperature of the growth surface; $F=(2+\cos \phi)(1-\cos \phi)^{2}$, where $\phi$ is the contact angle between the cluster and surface; and $\zeta=n / n_{\mathrm{e}}$ is the supersaturation determined by the ratio of the current, $n$, to equilibrium, $n_{\mathrm{e}}$, adatom concentrations.

First, according to relation (1), as the equilibrium is approached (as $n$ approaches $n_{\mathrm{e}}$ ), the sensitivity of the nucleation rate to the shape and surface energy of clusters increases strongly. An analysis of $F(\phi)$ also indicates that this stage of the cluster nucleation on the thin base layer should occur at $\phi<\pi / 2$. Obviously, this variant is possible when the area of the base layer reaches a certain value. Then, the $\phi$ value is observed to increase through increasing the cluster voluminosity. The latter assumption is supported by the fact that, at small variations in the spherical segment radius due to a voluminosity increase, the clusters gradually get darker in their color (see Figs. 1a and 1b).

It is known [16] that fcc metal nanoclusters may be formed on the basis of icosahedral, hexagonal, or fcc structures. In our case, electron diffraction studies (see Figs. 1 and 5) point to the nucleation of the nanoclusters with the fcc lattice whose parameters are close to the copper bulk state. This is obviously explained by the nucleation duplicating the structure of the thin base layer. The clusters with the fcc structure can be obtained on the crystal facets based on the (210) plane (see Fig. 2). The minimization of the free energy, in this case, is determined by the rounded shapes and the strongest chemical bonds for the atoms in the semicrystal position on the (210) plane [14]. At the same time, a pronounced nonmonotonicity of the dependence of the surface energy density of the clusters on the number of atoms in the clusters $[16,17]$ can reduce the work of their formation at a certain sequence of the incorporation of atoms.

As the condensation time increases under the technological conditions of the previous experiment, the area of the thin base layer increases (see the arrow directions in Fig. 1b). This is attributed to a further increase in the anion vacancy concentration. Taking into account that the condensation process is rather slow and analyzing the dynamics of varying the disper-

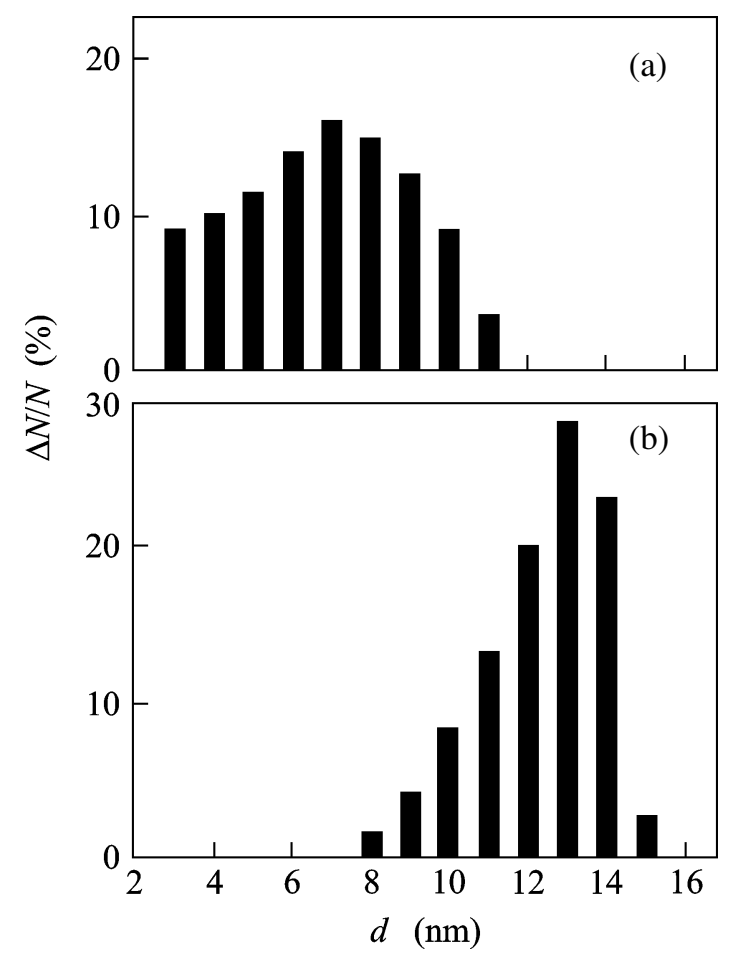

Fig. 3. Size distribution of the islands: $(a, b)$ histograms relating to the structures shown in Figs. 1a and 1b, respectively.

sion of the cluster structures (see Figs. 1a and 1b) and the corresponding histograms (see Figs. $3 a$ and $3 b$ ), we can conclude that the statistic uniformity of the Ostwald ripening is important for the self-organization. In this case, the nanocluster size distribution as a result of the Ostwald ripening agrees well with the theoretical notions [18], and the standard deviation from the average intercluster distance decreases from 45 to $18 \%$ during the process of cluster growth (see Figs. $3 c$ and $3 d$ ). An additional factor supporting the Ostwald ripening is a decrease in the concentration of clusters during the formation process by about $10-17 \%$. However, we should note that the Ostwald ripening, in our case, is a consequence not only of the decrease in the supersaturation and the corresponding growth of the critical nucleus size [19], but also of the stimulating effect on the growth surface of the condensed atoms and secondary electrons of increased energies. In this connection, the switching off of the magnetron sputterer should lead to a certain freezing of the Ostwald ripening process.

Even at a small increase in the discharge power, the cluster formation process is distorted by the transition to the epitaxial growth of sufficiently large islands (see Fig. 1c).

During the formation of the highly porous nanosystems, the specificity of the transition to the structure being a system of interlinked clusters is important. To understand the essence of the corresponding processes, 


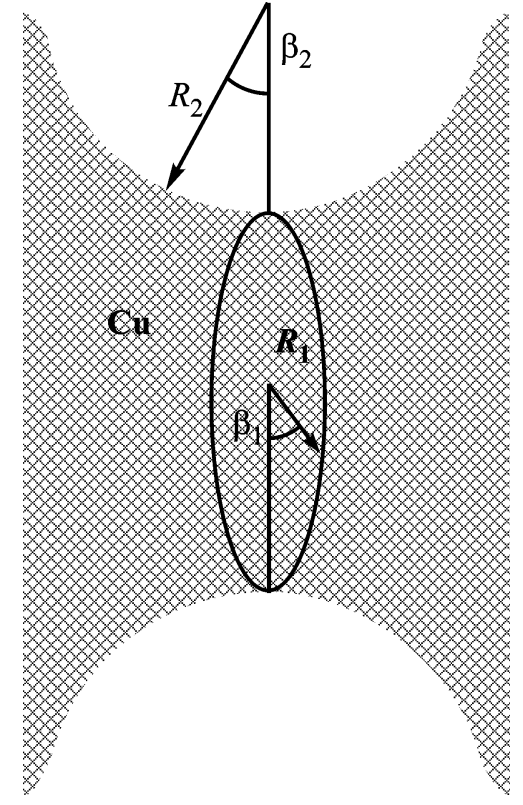

Fig. 4. Geometrical model of the coalescence region.

we consider a model of the coalescence region of two clusters (see Fig. 4). In the central part of the region, the equilibrium condition is determined by the Herring relation

$$
\mu_{c}-\mu_{k}=\frac{\Omega}{R_{1}}\left(\alpha+\frac{\partial^{2} \alpha}{\partial \beta_{1}^{2}}\right)-\frac{\Omega}{R_{2}}\left(\alpha+\frac{\partial^{2} \alpha}{\partial \beta_{2}^{2}}\right),
$$

where $\mu_{c}$ and $\mu_{k}$ are the chemical potentials of the vapor and condensate, respectively; $\Omega$ is the specific volume occupied by an atom in a crystal; and $R_{1}, R_{2}, \beta_{1}$, and $\beta_{2}$ are the radii of curvature and the corresponding angles in the coalescence region, respectively (see Fig. 4). In the simplified isotropic model, $\partial^{2} \alpha / \partial \beta_{1}^{2}=0$ and $\partial^{2} \alpha / \partial \beta_{2}^{2}=0$. In this case, if $R_{1}>R_{2}$, then equilibrium condition (2) is valid even for $\mu_{k}>\mu_{c}$. Hence, in the direct vicinity of the equilibrium, the instant of contact of the islands is a signal for the reorientation of the diffusion flows and for a faster growth of the condensate in the coalescence region. In this case, depending on the structural state of the contact surface, either the neck thickens (see Fig. 5a) or new clusters nucleate (see the arrow directions in Fig. 5b). The commonly known variant of the growth coalescence, in this case, is apparently impossible due to the ultimate minimization of the surface energy of the clusters. In other words, the initial instant of coalescence should be accompanied by a considerable increase in the surface energy, which is an insurmountable potential barrier for this process.

In some cases, due to the nonuniform distribution of the active centers over the substrate surface, a pronounced prevailing growth of the regions with an
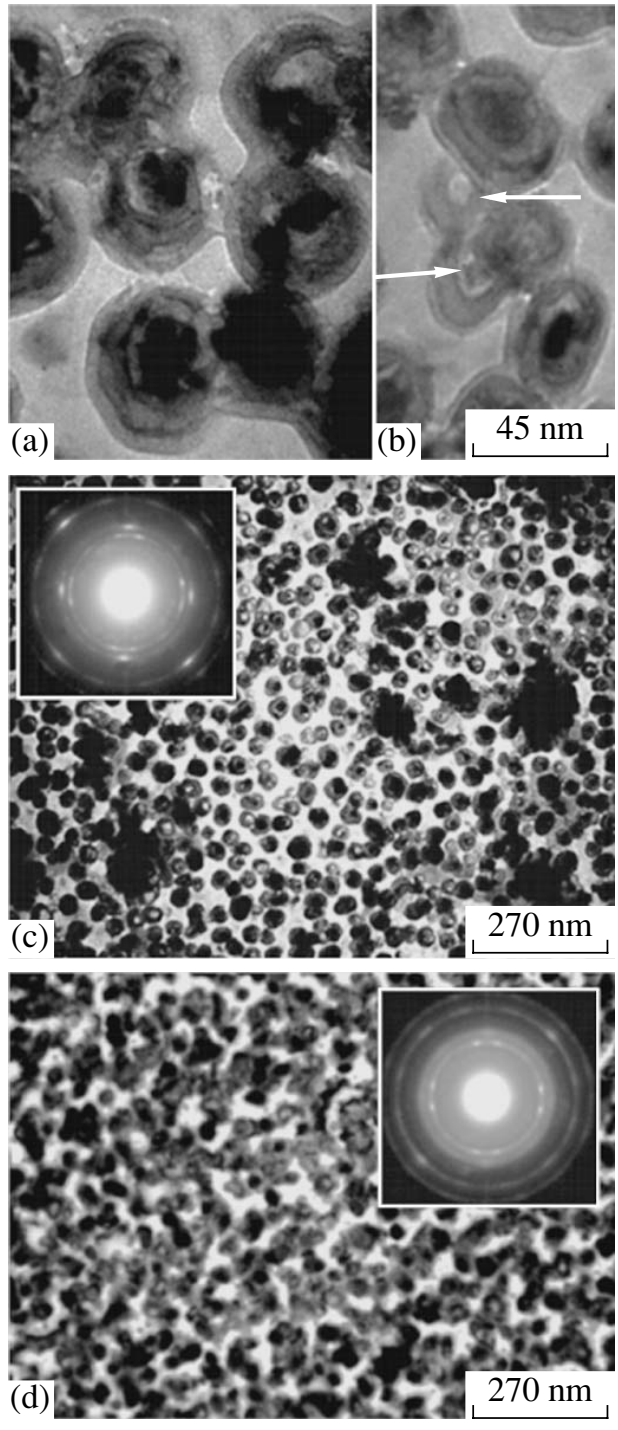

Fig. 5. Variants of the formation of nanosystems after 20-min condensation.

increased density of the clusters in contact with each other can be observed (see Fig. 5c). In this case, the redistribution of the diffusion flows blocks the growth of the other condensate regions. Meanwhile, a more uniform distribution of clusters, as well as the specificity of the prevailing substance condensation on the boundary of their coalescence, determines the formation of the 3D fractal networks (see Fig. 5d).

In summary, we have established that modern conventional notions of the metal condensation mechanism under Volmer-Weber conditions are limited only by sufficiently high supersaturations and that the cluster nanosystems can be formed under the conditions closest to the equilibrium ones. In this case, the near-equilibrium condensation and the Ostwald ripening, which are maintained by the effect of the particles on the growth surface, are the necessary prerequisites of the 
self-organization of the statistical uniformity of nanosystems, while the transition to the formation of fractal networks is conditioned by the secondary nucleation of the nanoclusters and the prevailing condensation in the region of their coalescence.

\section{REFERENCES}

1. O. P. Pchelyakov, Yu. B. Bolkhovityanov, A. V. Dvurechenskii, et al., Thin Solid Films 367, 75 (2000).

2. L. S. Palatnik, M. Ya. Fuks, and V. M. Kosevich, Mechanism of Formation and the Substructure of Condensed Films (Nauka, Moscow, 1972), p. 318 [in Russian].

3. V. I. Perekrestov, S. N. Kravchenko, and A. V. Pavlov, Fiz. Met. Metalloved. 88 (5), 72 (1999) [Phys. Met. Metallogr. 88, 482 (1999)].

4. V. I. Perekrestov, A. V. Koropov, and S. N. Kravchenko, Fiz. Tverd. Tela (St. Petersburg) 44, 1131 (2002) [Phys. Solid State 44, 1181 (2002)].

5. V. I. Perekrestov, Pis'ma Zh. Tekh. Fiz. 31 (19), 41 (2005) [Tech. Phys. Lett. 31, 830 (2005)].

6. V. I. Perekrestov and A. V. Pavlov, Pis'ma Zh. Éksp. Teor. Fiz. 73, 17 (2001) [JETP Lett. 73, 13 (2001)].

7. V. I. Perekrestov and Ya. A. Kosminskaya, Pis'ma Zh. Éksp. Teor. Fiz. 78, 258 (2003) [JETP Lett. 78, 223 (2003)].
8. S. A. Kukushkin and A. V. Osipov, Usp. Fiz. Nauk 168, 1083 (1998) [Phys. Usp. 41, 983 (1998)].

9. H. S. Nalwa, Handbook of Nanostructured Materials and Nanotechnology (Academic, Boston, 2000).

10. V. I. Perekrestov and S. N. Kravchenko, Prib. Tekh. Éksp., No. 3, 123 (2002) [Instrum. Exp. Tech. 45, 404 (2002)].

11. B. S. Danilin, Application of Low-Temperature Plasma for Thin-Film Deposition (Energoatomizdat, Moscow, 1989) [in Russian].

12. F. O. Goodman, Phys. Chem. Solids 23, 1269 (1962).

13. F. O. Goodman, Phys. Chem. Solids 24, 1451 (1963).

14. A. A. Chernov, Crystallization Processes (Nauka, Moscow, 1980) [in Russian].

15. G. I. Distler, J. Gryst. Growth 3, 175 (1968).

16. C. P. Poole, Jr. and F. J. Owens, Introduction to Nanotechnology (Wiley, Hoboken, N.J., 2003; Tekhnosfera, Moscow, 2006).

17. B. V. Smirnov, Physics of the Fractal Clusters (Nauka, Moscow, 1991) [in Russian].

18. R. D. Vengrenovich, Yu. V. Gudyma, and S. V. Yarema, Fiz. Tekh. Poluprovodn. (St. Petersburg) 35, 1440 (2001) [Semiconductors 35, 1378 (2001)].

19. S. A. Kukushkin and A. V. Osipov, Zh. Éksp. Teor. Fiz. 113, 2193 (1998) [JETP 86, 1201 (1998)].

Translated by E. Perova 